\title{
In older patients with late stage cancer, specialised home care by nurses improved survival after surgery
}

McCorkle R, Strumpf NE, Nuamah IF, et al. A specialized home care intervention improves survival among older post-surgical cancer patients.J Am Geriatr Soc 2000 Dec;48:1707-13.

\section{QUESTION: In older patients, does specialised home care by advanced practice nurses (APNs) increase survival after cancer surgery more than standard care?}

Design
Randomised \{allocation concealed\}*, blinded \{outcome
assessors\} $\}^{*}$ controlled trial with follow up of 44 months.

Setting

An urban academic health centre in Philadelphia, Pennsylvania, USA.

\section{Patients}

375 patients ( $52 \%$ women) who were $\geqslant 60$ years of age, diagnosed with a solid tumour $\leqslant 2$ months before surgical removal, and had an anticipated survival of $\geqslant 6$ months after surgery. Exclusion criteria were noncancer related surgery, discharge to an institution, or no baseline data before discharge. Follow up was complete.

\section{Intervention}

190 patients were allocated to specialised home care. APNs provided comprehensive clinical assessments, monitoring, and teaching to patients and their family caregivers over a 4 week period. Nurses made 3 home visits and 5 telephone calls. Nurses followed guidelines to monitor the physical, emotional, and functional status of patients; provided direct care; assisted in obtaining services from the community; and provided teaching, counselling, and support during the recovery period. 185 patients were allocated to standard care consisting of postoperative care in the hospital and routine follow up in outpatient clinics after discharge.

\section{Main outcome measure}

Length of survival.

\section{Main results}

By 44 months, 41 patients (22\%) in the home care group and 52 patients $(28 \%)$ in the standard care group had died. Patients receiving home care lived longer than those receiving standard care (hazard ratio [HR] 2.04, $95 \%$ CI 1.33 to 3.12, $\mathrm{p}=0.001$, adjusted for stage of disease at diagnosis and total length of stay in hospital for surgery) (table). Although patients with late stage cancer had a greater risk for death (adjusted HR 4.55, CI 2.92 to $7.08, \mathrm{p}<0.001$ ), home care improved their 2 year survival $(\mathrm{p}<0.05)$. Home care did not improve 2 year survival for patients with early stage cancer $\{p=0.93\}^{*}$.

\section{Conclusions}

Older patients with late stage cancer who received specialised home care from nurses had higher survival rates than patients who received standard care. Survival rates did not differ between care groups for patients with early stage cancer.

*Information/data provided by author.
Specialised home care $v$ standard care for survival in older patients at 44 months after cancer surgery

\begin{tabular}{|c|c|c|c|c|}
\hline Outcome & $\begin{array}{l}\text { Home } \\
\text { care }\end{array}$ & $\begin{array}{l}\text { Standard } \\
\text { care }\end{array}$ & RBI $(95 \% \mathrm{Cl})$ & NNT (Cl) \\
\hline Survival & $78 \%$ & $72 \%$ & $29 \%(13$ to 36$)$ & 5 (4 to 11$)$ \\
\hline
\end{tabular}

\section{COMMENTARY}

Age is a major risk factor for cancer, with more than half of new cases occurring in people over age 65 . This study by McCorkle $e t a l$ is the first to examine the effects of a specialised homecare intervention on the survival of older, post-surgical patients with cancer. Patients in the intervention group had a clinically important improvement in survival compared with those in the control group; in particular, patients in the intervention group who had late stage tumours lived almost twice as long as similar patients in the control group. The groups did not differ for quality of life measures such as depression, symptom distress, and physical and social functioning, suggesting that the increased survival is not at the expense of quality of life. Although the underlying mechanisms of action are not known, 3 factors may help to explain the results, and need to be studied. Firstly, APN availability through home visits, phone calls, and a 24 hour paging system may have permitted earlier detection and treatment of problems and complications. Secondly, the large educational component of the intervention provided by APNs may have led to earlier notification of problems. Finally, APNs may have helped patients and caregivers to develop a sense of control, which may have enabled them to engage in better self care activities.

The standardised protocol included guidelines for assessment and management, explicit instructional content, and a schedule for contact. Therefore, the intervention could serve as a model for implementation by community and home care nurses caring for older postoperative cancer patients. Further details of the educational component are reported in Hughes et al. ${ }^{1}$

Study patients were well educated, had good support systems, and most had minimal functional deficits before surgery. Replication across socioeconomic strata is warranted to determine generalisability of the study findings. Furthermore, the cost effectiveness of this intervention should be examined.

Sandra L Cromwell, RN, PhD Assistant Professor University of Arizona College of Nursing Tucson, Arizona, USA

1 Hughes LC, Hodgson NA, Muller P, et al. Information needs of elderly postsurgical cancer patients during the transition from hospital to home. J Nurs Scholarsh 2000;32:25-30. 